\title{
6 Unsere Zukunft im neuen Weltbild
}

\author{
Hans-Peter Emil Dürr
}

Ich habe als Physiker fünfzig Jahre lang - mein ganzes Forscherleben - damit verbracht, zu fragen was eigentlich hinter der Materie steckt. Das Endergebnis ist ganz einfach: Es gibt im Grunde keine Materie! Ich habe somit fünfzig lahre an etwas gearbeitet, was es gar nicht gibt. Das war eine erstaunliche Erfahrung: Zu lernen, dass es das, von dessen Wirklichkeit alle überzeugt sind, am Ende gar nicht gibt. Immerhin hat es sich gelohnt, diesen langen Weg zu gehen. Wir werden dann die Frage stellen, was für uns daraus folgt, welche Konsequenzen wir daraus für die jetzige Situation ziehen können, die so voller Krisen ist. Denn diese Krisen hängen alle damit zusammen, dass wir eine völlig falsche Vorstellung von der Welt haben. Wir haben uns in ein enges Weltbild hineindrängen lassen, in dem es keine Lösungen gibt. Aus diesen Fesseln eines engen Weltbildes müssen wir herauskommen.

Was uns alle täglich bedrängt, unsere zentrale Aufgabe, ist die Frage der Zukunftsfähigkeit, eine Frage des Überlebens der Menschheit. Das ist auch, was man Nachhaltigkeit nennt, die gesellschaftliche Herausforderung unserer Zeit. Es geht um Zukunftsfähigkeit, die dann vorhanden ist, wenn wir die Fesseln unseres zu engen Weltbildes abstreifen. Wir brauchen dazu eine neue Orientierung und auch die Fähigkeit, zu handeln und zu gestalten. Diese Fähigkeit, Dinge verändern zu können, haben wir tatsächlich. Allerdings nicht mehr im Rahmen der alten Weltvorstellung, in der wir uns wie ein Rädchen in einer Maschine verstehen müssen, sondern wir haben wirklich schöpferische Fähigkeiten, und wir sollten sie auch gebrauchen.

Ein offenes Problem ist, dass wir dabei sind, unsere natürlichen Lebensgrundlagen zu zerstören. Viele glauben noch immer, dass die Menschheit gewissermaßen über der Natur schwebt, und dass wir machen können, was wir wollen. Sie sehen nicht, dass wir als Menschheit in ein größeres Ganzes eingebettet sind. Wenn wir dieses größere Ganze zerstören, sind wir die Ersten, die abstürzen werden.

Ein anderes Problem ist, dass Gerechtigkeit und Frieden heute weiter entfernt sind als je zuvor, und wir wissen nicht recht, wie wir da herauskommen. Die meisten halten Gerechtigkeit und Frieden für eine 
ganz irreale Vision. Sie glauben, dass die Wirklichkeit so ist, wie sie ist, und dass es am Schluss immer ungerecht und friedlos zugeht. Eine Natur, in der am Ende immer nur der Mächtigste überlebt, und die anderen einfach zugrunde gehen. Das sind aber grundfalsche Vorstellungen, die mit unserem falschen Menschenbild und Weltbild zusammenhängen.

Schwer wiegt auch der Verlust der geistigen Dimension. Viele wissen gar nicht mehr, was die geistige Dimension ist. Aber wir können - auch heute - in dieser Welt gar nicht von dem leben, was greifbar ist. Viele behaupten, sie wären ganz rational, sie glauben nur, was sie beweisen können. Aber es ist gar nicht möglich, auch nur eine Sekunde so zu leben! Viele glauben, sie hätten alles verstanden. Sie haben aber nichts verstanden - und trotzdem leben sie weiter, weil im Hintergrund doch etwas ist, das uns immer noch den Weg zeigt. Das Herz schlägt weiter, auch wenn man nicht daran glaubt, dass es schlägt.

\section{Neue Technologie - altes Denken}

Das nächste Problem ist eine Inkonsistenz zwischen Denkweise, Technologie und zukünftigen Erfordernissen. Wir haben eine Denkweise, die immer noch die alte ist - nämlich die des 19. Jahrhunderts - in der wir die Welt als eine materielle, mechanistische Maschine betrachten, ein mechanistisches und materialistisches Weltbild. Aber unsere heutige Technik ist auf einer ganz anderen Vorstellung aufgebaut, eben auf den Errungenschaften dieser neuen Betrachtung, die Physiker vor etwa hundert Jahren herausgefunden haben. Daraus folgt ein ganz anderes Weltbild. Die wirklich neue Denkweise hat zwar noch nicht Fuß gefasst, aber sie hat unsere Technik neu geformt. Wir haben heute nicht mehr Technik, sondern Technologie.

Was ist der Unterschied zwischen Technik und Technologie? ,Logie deutet auf eine Lehre, das heißt, dass man erst irgendetwas lernen und verstehen muss, bevor man es betreiben kann. Ja man bräuchte eine Lehre, aber es gibt noch keine Lehrer dafür. Das ist der Grund, warum wir uns nicht entschieden haben, darüber klärend nachzudenken, wie diese neue Technologie eigentlich funktioniert. Glauben Sie nur nicht, dass Sie verstehen, was da im Hintergrund passiert, wenn Sie ihren Computer benützen. Sie haben eine Vorstellung davon, aber eigentlich wissen Sie es nicht, denn Sie können es mit der alten Sprache nicht mehr benennen. Das heißt, wir haben heute eine neue Technologie, dazu gehört die moderne Chemie, die Mikroelektronik, und dazu gehört selbstverständlich auch die Entwicklung der Atombomben. Das ist alles Technologie, die nur mit dem neuen Denken funktioniert. Das ist uns aber nicht bewusst. Und jetzt wollen wir das 21. Jahrhundert gestalten - mit der modernen Technologie und der falschen Denkweise. Warum hängen wir so an diesem alten materialistischen, mechanistischen Weltbild? Weil es mit Händen greifbar, begreifbar ist. Materie ist etwas, das ich greifen kann. Materie ist etwas, das eine begrenzende Oberfläche hat. Ich kann es in der Hand halten und sagen, es ist meines. Es ist letztlich diese Materie, die uns in der industriellen Entwicklung dazu geführt hat, Dinge in dieser Welt nicht nur manipulieren, sondern auch besitzen zu können und damit zu einer verschiedenen Eigentumssituation zu gelangen.

Es liegt an überholten Weltbildern, die dazu führen, dass wir Menschen uns außerhalb der Natur empfinden, als etwas von der Natur Verschiedenes. Naturvergessenheit könnte man das nennen. Damit haben auch die Religionen zu tun. In der Art und Weise, wie der Mensch über die Natur erhoben und Gott ähnlich gemacht wird, haben wir die Natur erniedrigt. Und der Mensch betrachtet die Natur nur noch als Werkzeug oder als Bausteine und als nichts anderes - obwohl wir wissen, dass wir selbst 
in die Natur eingebettet sind. Diese Vorstellung der Trennung müssen wir überwinden. Das führt dazu, dass wir einerseits überschätzen, was wir alles machen können, und andererseits unterschätzen, was wir wirklich an Möglichkeiten haben. Die neuen Naturwissenschaften haben aufgezeigt, dass es nicht die strenge Naturgesetzlichkeit gibt, wie wir uns das früher vorgestellt haben. Hinter den Dingen steht eine Verbundenheit, die nicht streng ist, sondern eine gewisse Offenheit aufweist.

Wir haben somit eine Situation, die für uns viel günstiger ist als wir uns bisher vorstellen. Wir haben zwar einerseits Gesetzlichkeiten, aber andererseits eine Offenheit, die wir nützen können, um auch selbst Einfluss zu nehmen. Aber diese Offenheit ist gar nicht so einfach erlebbar. Sie hängt damit zusammen, dass wir, wenn wir handeln und agieren, immer von einem Hintergrund her handeln, der mit irgendeinem vagen Wissen zu tun hat, mit Ahnungen, aus denen heraus neue Ideen entstehen. Die Ahnung selbst ist in einer anderen Welt, als die Welt es ist, in der wir Dinge im wörtlichen Sinne begreifen und sagen können: „Das habe ich verstanden, ich halte es hier in der Hand. "In dieser anderen Welt erfahren wir diese Offenheit. Die neuen Einsichten zeigen uns, dass wir doch kreative Fähigkeiten haben. Es ist eine allgemeine Eigenschaft, und wir können darauf vertrauen, dass es notwendig und wichtig ist, diese Eigenschaft des Kreativen auch zu benutzen. Dann sehen wir auch, dass es mit in unserer Hand liegt, wie die Zukunft gestaltet wird. Zukunft ist nicht völlig fixiert durch Naturgesetzlichkeit. In diesem Zusammenhang können wir fragen:

- Welche Zukunft wollen wir?

Das ist keine sinnlose Frage, wir können die Zukunft wirklich gestalten, selbstverständlich nicht generell, aber als prinzipielle Möglichkeit. Es hat wenig Sinn zu warten, was die Zukunft bringt. Nein, wir müssen nicht warten, wir müssen uns überlegen, welche Zukunft wir wollen.

- Welche Vision haben wir von der Zukunft?

Wenn wir diese Vision haben, dann wissen wir auch, dass wir uns dieser Zukunft als einer Möglichkeit annähern können. Nicht allein, aber mit anderen zusammen.

Wir alle haben die Veranlagung zum „Homo sapiens sapiens“ - der weise Mensch und der ist nicht nur auf einige Menschen begrenzt. Wir geben uns überhaupt nicht die Mühe, den Menschen dorthin zu bringen. Wer spricht heute schon von Menschen? Wir sprechen von Arbeitsplätzen, aber nie von dem Menschen, der handeln will, der sein Leben gestalten will. Davon ist nicht die Rede. Nur von Arbeitsplätzen $\mathrm{zu}$ reden, das ist eine sterile Sprache, bei der man den Menschen, den eigentlichen Menschen, gar nicht mehr sieht. Statt dass die Technik dafür da ist, diesem Menschen zur Entfaltung zu helfen, laufen wir hinter der Technik her, damit wir den Anforderungen gerecht werden, die diese neue Technik an uns stellt. Das geht völlig am wirklichen Leben vorbei. Wir alle sind dieser „Homo sapiens sapiens“. Jeder von uns ist einmalig und kann einen Beitrag in diese Richtung leisten.

\section{Nachhaltigkeit}

Es geht um Zukunftsfähigkeit, um Nachhaltigkeit. Ich war selber daran beteiligt, dass dieser Begriff verwendet wurde. Im Frühjahr 1990 - eben war die Mauer gefallen - trafen wir, eine ökologische Gruppe aus der Bundesrepublik uns erstmals mit einer ökologischen Gruppe der damaligen DDR, darunter sehr viele Forstwirte, im 
bekannten Auerbachs Keller in Leipzig. Wir diskutierten eifrig über eine Reihe dringender ökologischer Probleme und dabei unter anderem auch allgemein über „sustainability“, für das wir noch keine gute deutsche Übersetzung hatten. Da kam der Vorschlag, warum nehmen wir nicht „Nachhaltigkeit“? Ein Begriff, der in der Forstwirtschaft verwendet wird: Man soll nicht mehr Holz schlagen, als nachwächst. Einige, wie auch ich, waren darüber nicht so glücklich, da dies ja schon aus rein wirtschaftlichen Gründen gefordert werden muss. Aber die Forstwirte betonten, dass in der Forstwirtschaft dieser Begriff eine umfassendere Bedeutung hat, die z.B. die Bodenqualität und Vielfalt in der Bepflanzung einschließt. Darauf haben wir uns dann geeinigt. Aber ich konnte mich mit diesem Ausdruck nie so recht anfreunden. Nachhaltigkeit ist ein so langweiliger Begriff, bei dem man gar nicht merkt, dass es sich dabei um etwas ganz Aufregendes handelt. Da ist „sustainability“ schon etwas besser, denn da ist eine „ability“, eine Fähigkeit drin. Aber „nach“ und „halten“? Meine Abneigung gegen diesen Begriff liegt auch daran, dass dabei nicht zum Ausdruck kommt, was eigentlich gemeint ist. Es bedeutet eben nicht, dass wir diese Welt, so erhalten wollen, wie sie jetzt ist, sondern wir wollen die in dieser Welt angelegte Dynamik, Vitalität und Produktivität bewahren und fördern. Die Robustheit und Elastizität wollen wir schon beibehalten, aber nicht den augenblicklichen Zustand. Es soll in dieser Richtung, mit dieser Lebendigkeit weiter gehen.

Das bedeutet aber auch, dass wir nie nur den Menschen allein in diese Nachhaltigkeit hineinnehmen können. Nachhaltigkeit bezeichnet die Lebendigkeit des ganzen Biosystems, in dem wir eingebettet sind. Dabei können wir drei Ebenen unterscheiden:

1. Wir wollen die natürlichen Lebensgrundlagen nicht zerstören,

2. wir wollen auch, dass die Menschen friedlich und in Gerechtigkeit zusammenleben und

3. wir wollen auch ein gutes und lebenswertes Leben haben.

Das imitiert ein bisschen die 3-Säulen-Theorie: Nachhaltigkeit/Ökologie, Gesellschaft und Ökonomie. Nur dass Ökonomie nicht die volle Entfaltung des Menschen als „Homo sapiens sapiens“" charakterisiert, sondern nur seine materiellen Grundlagen. Es geht aber nicht nur um das physische Überleben des Menschen, sondern auch darum, dass er seine emotionale und geistige Potenzialität, das seine Persönlichkeit formt und seine Eigenart ausmacht, optimal entwickeln kann. Jeder hat dazu die Gabe und kann dies für sich fordern. Es bezeichnet eine wesentliche Voraussetzung für den Menschen, die durch die Ökonomie nicht abgedeckt werden kann.

Völlig verkehrt ist es, wenn man zwar von diesen drei Säulen ausgeht, aber die Ökonomie dann an die erste Stelle setzt, weil man glaubt, zunächst für optimale Wettbewerbsverhältnisse sorgen zu müssen. Sodann wird an zweiter Stelle die Aufmerksamkeit auf die gesellschaftlichen Probleme gerichtet und schließlich ganz am Schluss, soweit noch genügend Kraft vorhanden, will man sich, gleichsam im Sinne einer Dekoration, auch noch um die Natur kümmern.

Nein, so geht dies nicht. Die Prioritätenfolge muss genau umgekehrt sein. Die Natur, die natürliche Lebensgrundlage, ist das Fundament, in dem die Menschheit als Spezies existentiell eingebettet ist. Wir müssen dafür sorgen, dass die natürlichen Lebensgrundlagen nicht zerstört werden, und dann auch, dass sich die Menschheit in dieser Welt mit gesunden Lebensgrundlagen entwickeln kann, und dass der Mensch in der Gesellschaft nicht nur als ein Rädchen zählt, sondern sich als Individuum in seiner Eigenart entfalten kann und als verantwortlicher Träger der Gemeinschaft sei- 
nen angemessenen Platz in dieser findet. Das heißt, die System-Hierarchie, wie sie üblicherweise immer aufgezeigt wird - Ökonomie an erster, Gesellschaft an zweiter und Ökologie an dritter Stelle - muss genau umgedreht werden. Der Mensch ist der sensibelste Teil des Ökosystems. Wenn etwas schief läuft, ist er der erste, der abstürzt.

\section{Wir betreiben nicht Ökologie, weil wir einfach die Schönheit der Natur lieben, sondern weil unsere Existenz auf ihrer Existenz beruht.}

\section{Elemente des neuen Denkens}

Nach der alten Weltsicht ist die Welt etwas Äußeres. Ich schaue die Welt da draußen an, und was ich wahrnehme, ist hauptsächlich Materie. Deshalb nennen wir diese Welt „Realität“, vom lateinischen „res“, das Ding. Die Welt besteht aus Materie und ist etwas, das ich begreifen kann. Wenn ich sage, die Welt ist „Realität“, sage ich gleichzeitig: Die Welt kann begreifbar sein. Ich kann etwas in die Hand nehmen und sagen, ich habe ein Stück dieser Welt in der Hand. Die Anordnung der Materie in der Zeit geschieht mit einer gewissen Regelmäßigkeit. Wir haben festgestellt, es gibt streng geltende Naturgesetze. Daher können wir von der jetzigen Konfiguration ausgehend sagen, wie die Anordnung im nächsten Augenblick sein wird, und so weiter. Sowie auch rückwärts. Mit dieser Naturgesetzlichkeit kann gewissermaßen gezeigt werden, was in Zukunft passiert oder was in der Vergangenheit passiert ist. Das ist auch tatsächlich möglich, und daher haben wir den Eindruck, wir bekommen die Welt in den Griff.

Die neue Wirklichkeit ist aber ganz anders, als wir sie uns bisher vorgestellt haben: Materie ist im Grunde nicht Materie. Deshalb habe ich eingangs erwähnt, ich habe fünfzig Jahre über Materie gearbeitet, die es gar nicht gibt. Wir können uns das nicht vorstellen. Es ist, als ob man sagt, am Anfang gibt es nur Software und gar keine Hardware. Eine Software, die man nicht begreifen kann, die nur eine Gestalt, aber keine Existenz im ursprünglichen Sinne des Wortes hat. Es gibt nur eine Beziehungsstruktur, es gibt keine Objekte. Die Frage, was ist und was existiert, kann nicht mehr gestellt werden.

Es gibt nur noch innere Form oder Gestalt. Das ist für die Alltagsvorstellung unverständlich. Für uns ist die Form eine Anordnung irgendeiner Substanz. Aber alle erleben wir heute eine Welt, bei der wir von einer eingeprägten Form, einer Gestalt ohne Substanz reden können.

Wie erklären Sie jemandem, was da vor sich geht, wenn Sie mit dem Mobiltelefon mit Freunden in Paris telefonieren?

„Ich habe da so eine Antenne dran, und irgendwie kitzelt diese Antenne da so ein Feld im Hintergrund, und das gibt dann eine Welle im Äther. Diese Welle im Äther kann mein Freund in Paris empfangen und das ist das Gespräch."

Das wäre fast richtig, nur - es gibt den Äther nicht. Das heißt, die Welle hat keinen materiellen Träger. Du hast das Nichts gekitzelt, und damit eine „Delle“ in diesem Nichts erzeugt. Diese Delle ist eine reine Form des Nichts. Dein Freund in Paris emp- 
fängt diese Delle und sagt, da kommt mein Gespräch. Dabei ist aber nichts Materielles passiert. Und das ist der Grund, warum Sie ihr Handy an jedem Ort benützen können. Sie können das Nichts kitzeln, wo sie wollen, das spielt gar keine Rolle. Ihr Freund in Paris kann sein, wo er will, er kann die Delle überall wahrnehmen.

Mit diesem Beispiel bekommen Sie schon ein Gefühl, dass das Materielle und seine Lokalisierung keine primäre Rolle mehr spielt. Wir arbeiten mit einer reinen Gestaltstruktur. Das macht es natürlich nicht unbedingt anschaulicher, sondern eher kompliziert und unverständlich. Diese Gestalt hat keinen Ort, an dem sie sich befindet. Die Gestalt ist sozusagen über die ganze Welt ausgebreitet. Es gibt überhaupt keine Auflösung in Teile. Das heißt, in der Physik ist die Wirklichkeit nicht Realität, sondern Potenzialität. Sie ist nur die „Möglichkeit“, die sich energetisch und materiell irgendwo manifestieren „kann“, sozusagen etwas noch nicht Entschiedenes, Schwebendes. Und diese Potenzialität ist räumlich nicht lokalisiert. Die Welt ist das Eine und Ganze. Das führt dazu, dass die ganze Welt überhaupt keine Ränder hat. Es gibt nur das Eine, und wir könnten sagen, es ist das Ganze. Das „Ganze“ ist aber auch nicht das richtige Wort. Das Ganze ist ja etwas, dem kein Teil fehlt. Aber wenn es gar keine Teile gibt, dann können wir es auch nicht das Ganze nennen. Aber stellen Sie sich etwas vor, das man überhaupt nicht zerlegen kann. Das hat selbstverständlich phantastische Konsequenzen. Das heißt zum Beispiel, wenn wir uns selbst wirklich in diese Welt einbeziehen, dass wir alle zwar unterschiedlich und unterscheidbar, aber nicht getrennt sind. Wir befinden uns alle sozusagen in dieser Gemeinsamkeit, und das ist eine wesentliche Voraussetzung, dass wir überhaupt miteinander kommunizieren können.

Die Welt ist das Eine und Ganze. Im Sanskrit nennt man das „Advaita“, A-Dualität. Das bedeutet: etwas, das man überhaupt nicht aufteilen kann, wo die Zerstückelung erst gar nicht möglich ist.

Der entscheidende Schritt, in dem wir festgestellt haben, dass die Materie verschwindet und die Form bleibt, hat mit der Struktur des Atoms zu tun.

Warum haben wir die Atome erforscht?

Wir wollten die Welt in den Griff bekommen, und wir wussten, dass uns das gelingt, wenn wir die Materie von der Form ablösen können.

Aber wie ist das möglich?

Ich muss die Form beseitigen und nehme dazu ein Beil, zerhaue beispielsweise diesen Tisch, dann ist seine Form kaputt. Aber nicht generell; es entstehen daraus zwei Teile, die wieder je eine Form haben. Daher verwende ich das Beil wieder und wieder in der Hoffnung letztlich jegliche Form zu beseitigen. So wird man zum Atomphysiker. Beim Atom angekommen, meinten wir endlich am Ziel zu sein. Jetzt haben wir das a-tomos, gefunden, was sich nicht mehr spalten lässt. Es ist reine Materie ohne Form. Doch dann kam Lord Rutherford und zeigte, dass auch dieses Atom noch eine Struktur hat. Wieder musste man das Beil nehmen und nachsehen, wie es im Inneren des Atoms ausschaut. Man fand eine Struktur, die einem Planetensystem glich mit einem schweren Kern und, diesen umkreisend, leichteren Elektronen, zusammengehalten jedoch durch elektrische Kräfte. Es erschien leicht, es in Analogie zu unserem bekannten gravitativ zusammengehaltenen Planetensystem zu verstehen 
und mechanistisch zu erklären. Doch dies misslang: Das elektrische Planetensystem funktioniert nicht: Es ist nicht stabil. Und jetzt ist es passiert: Es widerspricht den Naturgesetzen, so wie wir sie kannten!

Die Naturgesetze der Mechanik sind so einfach, dass uns nicht die Ausrede bleibt, wir haben das System noch nicht ganz verstanden und werden schon irgendwann eine Lösung finden. Es bleibt nur die Folgerung:

Die bisherigen Naturgesetze sind im Grunde falsch und - welche Überraschung - wir müssen feststellen, es gibt die Materie im Grunde nicht mehr. Es gibt letzten Endes nur noch eine Art Schwingung. Es gibt streng genommen keine Elektronen, es gibt keinen Atomkern, sie sind eigentlich nur Schwingungsfiguren. Eine Art Schwingungsfigur wie ein Handy-Gespräch im elektromagnetischen Feld, nichts Materielles im eigentlichen Sinne. An diesem Punkt haben wir die Materie verloren.

- Welche Konsequenz hat das?

- Haben diese überraschenden und paradox erscheinenden neuen Einsichten in der Mikrophysik überhaupt eine Konsequenz für uns in unserer viel größeren Lebenswelt?

Der entscheidende Punkt in unserem Fall ist nun der folgende: Wenn ich in unsere Welt aufsteige, in der Billionen mal Billionen Atome in einem Gramm Materie versammelt sind, dann rede ich nicht mehr nur von einem einzelnen Atom. Eigentlich sollte ich überhaupt nicht vom „A-tom“ oder einem Mikro-Teilchen sprechen, das gibt es ja gar nicht mehr. Ich sollte vielmehr von der Vorstellung eines „Passierchens“- etwas, das passiert, etwas Prozesshaftes - oder eines „Wirks“, einer kleinen Wirkung, ausgehen. Das deutsche Wort „Wirkung“ für die Wirklichkeit ist ja viel besser als das der „Realität“. Ich nehme also einen Sack von Billionen mal Billionen Passierchen, schüttle ihn durch und frage: Wie verhält sich dieser Sack insgesamt? Es wird etwas Gröberes, undifferenzierteres Durchschnittliches sein. Und siehe da, was dabei herauskommt - nicht ganz überraschend aber zu unserer großen Erleichterung -, ist genau die alte Physik: die Naturgesetze, die Kausalität, die Welt. Alles ist auf einmal mit großer Bestimmtheit da, nicht absolut scharf, sondern mit kleinen Abweichungen. Wenn ich Billionen mal Billionen Atome habe, ist die Abweichung plus/minus ein Billionstel. Das beunruhigt uns selbstverständlich nicht. Aber könnte es doch vorkommen, dass diese Passierchen, diese kleinen Prozesse, sich nicht ausmitteln, dass sie gewissermaßen nicht notwendig total ungeordnet durcheinander gewürfelt sind? Könnte es sein, dass sie in ihrer Gesamtheit eine gewisse Ordnungsstruktur beibehalten, sodass das, was im Mikroskopischen angelegt ist, doch verstärkt nach oben steigt und in unserer Lebenswelt zum Ausdruck kommt?

Betonen möchte ich auch, dass wir etwas, das fundamental kreativ und indeterminiert angelegt ist, eigentlich nicht mit dem „toten“ Materiellen vergleichen sollten. Denn es hat die wesentlichen Ingredienzien dessen, was wir lebendig nennen. In gewisser Weise können wir deshalb sagen: Im Urgrund oder an der Urquelle ist etwas, das dem Lebendigen viel ähnlicher ist als der Materie. Es ist nämlich alles im ewigen Wandel, und es ist nur der Wandel, der sozusagen das Bauelement ist und nicht ein Etwas, was verwandelt wird. Das Etwas gibt es gar nicht. Die Materie kommt auf 
einer Ebene zustande, wo sich alles Wandelnde überlagert, wie Ameisen eines Ameisenhaufens im Wald. Dieser Ameisenhaufen steht einfach als Kegel da, obwohl die Ameisen alle durcheinanderlaufen. Obwohl alles in Bewegung ist, bleibt etwas im Mittel stehen wie ein Erdkegel - aber nur in der Vergröberung. Könnte es sein, dass dieses Gewusel sich auf einmal so anordnet und ordnet, dass es zu dem führt, was wir in unserer Lebenswelt Leben nennen? Dann müssen die Passierchen verstärkt, muss das Kleine sozusagen vergrößert werden. Wie wenn ich den Ameisenhaufen mit einem Vergrößerungsglas betrachte und bemerke, das sind ja eigentlich Ameisen.

\section{Das Paradigma des Lebendigen}

Warum laufen wir auf zwei Beinen? Wenn wir auf einem Bein stehen, ist das eigentlich instabil. Es wäre doch viel besser, auf vier Beinen zu stehen, immer in Sicherheit. Ein Bein gibt uns aber das Erlebnis, frei zu sein. Ich kann in jede gewünschte Richtung fallen, ein Glücksgefühl sozusagen. Ein zweites Bein hat auch dieses Glücksgefühl. Aber jedes Bein hat nur einmal die Chance. Doch es gibt einen Ausweg. Wenn die beiden Beine geeignet kooperieren und es ihnen gelingt, im Wechseltakt hintereinander diesen Punkt zu erreichen, also mein zweites Bein genau dann nach vorne schwingt, wenn ich auf dem ersten umfalle - und umgekehrt, dann falle ich nicht mehr, sondern gehe beschwingt dahin. Ich habe meine beiden instabilen Systeme dynamisch stabilisiert. Zwei instabile Systeme, die sozusagen das Entgegengesetzte machen, sind in diesem Falle keine Gegner sondern Kollaborateure. Zusammen können sie ein Spiel inszenieren, das zu einer Bewegung führt, mit der ich kilometerweit durch die Gegend laufen kann, ohne zu fallen. Jetzt erst wird das Leben lebenswert, weil ich es so lange aufrechterhalten kann.

Aber ich habe in diesem Beispiel etwas Wesentliches unterdrückt, das ich notwendig noch hinzufügen muss. Gehen ist nicht nur ein kooperierendes, ewiges Fallen, sondern bei jedem Schritt nach vorne muss das nach vorne geschwungene Bein etwas in die Knie gehen und sich wieder strecken. Ich muss ihm Energie zuführen, um nicht am Ende doch am Boden zu landen.

\section{Leben muss gefüttert werden!}

Das ist der Grund, warum wir essen müssen. Durch energie-unterstützte dynamische Stabilisierung von Instabilitäten haben wir also die Möglichkeit eine hohe Sensibilität, die uns in reichem Maße die Welt öffnet, lange Zeit aufrechtzuerhalten und damit einen möglichen Zugang, was wir in unserer Sprache als Lebendigkeit betrachten und erfahren. Die dynamische Stabilisierung ermöglicht eine stetige Höherentwicklung des Lebendigen auf unserer Erde. Die Sonne liefert uns dazu gewissermaßen die Energie. Aber nicht nur uns, alle lebenden Systeme in der Welt werden vom Sonnenlicht genährt. Genau betrachtet ist es nicht ganz richtig zu sagen, es sei die Energie der Sonne, die eine Rolle spielt. Die von der Sonne eingestrahlte Energie wird ja wieder in den Weltenraum zurückgestrahlt, sonst würden wir hier auf der Erde allmählich ins Sieden kommen. Es geht vielmehr nur um eine Ordnungseigenschaft, die Syntropie, die im aufgenommenen Sonnenlicht höher ist als in der abgegebenen Wärmestrahlung, von der alles Lebendige abhängt. Wir haben hier im Lebendigen einen interessanten Prozess. Ich möchte es das „Paradigma des Lebendigen“ nennen, 
das ganz andersartig ist als das „Paradigma des Unlebendigen“. Letzteres erlebt man jeden Tag an seinem Schreibtisch. Wenn man den ganzen Tag herumhantiert, wird der Schreibtisch immer nur unordentlicher und nie ordentlicher. Das ist der zweite Hauptsatz der Thermodynamik oder, direkt einsichtig, die Aussage: In Zukunft passiert das Wahrscheinlichere wahrscheinlicher. Oder anschaulicher: Ein geordneter Schreibtisch ist eine unwahrscheinliche Konfiguration. In Zukunft wahrscheinlicher ist der unaufgeräumte Schreibtisch. Wenn er total unordentlich ist, dann kann man noch stundenlang darin wühlen, und er wird nicht noch unordentlicher. Das nennt man den thermodynamischen Grundzustand, weil dem alles zustrebt. Das ist der natürliche Endzustand. Aber wir, die wir am Wochenende den Schreibtisch mit ordnender Hand aufräumen, um wieder erledigt und unerledigt zu trennen oder Papiere anders zu ordnen, brauchen dazu Energie. Dabei ahmen wir gewissermaßen die Sonne nach. Aber wir brauchen auch eine Sensibilität, um erst das anzuschauen und zu bewerten, was wir ordnen wollen. Im Lebendigen ist es dieser Prozess der ordnenden Hand, der zu einer Differenzierung führt. Das ist der lebenden Natur von vornherein eingeprägt oder von der überlebenden erlernt, dass alles danach strebt, sich zu unterscheiden, d.h. in verschiedenen Formen vorzukommen. Aber diese Differenzierung bedeutet nicht, dass wir getrennte Dinge bekommen, sondern alles verbleibt noch im Ganzen verknüpft. Es ist so wie in einer Familie, wo jeder innerhalb eines Hauses ein anderes Zimmer aufsucht, aber keiner das Haus verlässt. Das heißt, für die Differenzierung brauche ich eine Neuschöpfung, Kreativität, die Fähigkeit aus der gewohnten Reihe zu tanzen. Dies reicht jedoch nicht aus. Es muss sich daran ein kreativer Prozess anschließen, bei dem, was unterschiedlich ist, ein kooperatives Spiel beginnt, das eine organismische Integration erlaubt.

Wie können wir miteinander leben und zu einem neuen Ganzen finden? Dies erfordert ein Plus-Summen-Spiel, wo man die spezielle Eigenart nicht aufgibt, sondern jeder seine Unterschiedlichkeit behalten kann - wie bei einem Orchester ein Konzert gelingt, bei dem verschiedene Instrumente konstruktiv zusammenspielen, und so das Ganze mehr wird als die Summe der Teile. Das ist das Paradigma des Lebendigen. Der erste Prozess ist die Individualisierung des Menschen, seine Emanzipation, und die müssen wir unterstützen. Wir wollen, dass die Menschen verschiedenartig sind. Denn wenn ich nur ein Bein habe, das an das andere geschnürt ist, falle ich genauso schnell wie mit einem Bein. Wir müssen zunehmend verschieden werden, damit wir immer mehr Stützfunktionen übernehmen können, die immer mehr dynamische Stabilisierungen ermöglichen. Erst dann können wir auf verrückte Weise durch die Welt gehen, ohne zu fallen. Die Höherentwicklung des Lebendigen durch Kombination aus Differenzierung und das kooperative Zusammenspiel von Verschiedenartigem ergibt eine neue Ganzheit, ein neues „Holon“. Auch der Mensch ist so ein Ganzes, das eine gewisse Abgeschlossenheit hat. Aber wenn Sie sehen wie viel Gegensätzliches in uns wirkt, auf wie vielen Ebenen wir immer wieder dieses Gleichgewicht herstellen müssen, dann offenbart sich hier eine hoch-integrierte, eine globale Struktur des Systems.

Globalisierung ist also an sich nichts Schlechtes, im Gegenteil es ist eine Notwendigkeit, um eine höhere Entwicklungsstufe zu erklimmen, aber es bedeutet zwingend, dass die Verschiedenartigkeiten ein Plus-Summen-Spiel inszenieren müssen, das sich nicht nur als miteinander verträglich erweist, sondern wo auch der Vorteil des einen im Durchschnitt zum Vorteil der anderen gereicht. Es kann nicht eine Gruppe von Menschen sagen, diese oder jene Eigenschaft ist wichtiger oder wertvoller und soll deshalb globalisiert werden und alles Übrige unterdrückt und als Störenfried be- 
handelt wird. Wir müssen dafür sorgen, dass alle Kulturen dieser Welt in ihrer Substanz bestehen bleiben und in die angestrebte überwölbende Kultur hineingenommen werden. Denn nur die Summe aller dieser Kulturen kann in diesem Zusammenspiel eine Weltkultur schaffen, die ein neues „Holon“ bildet, das eine höhere Entwicklungsstufe ausweist. Wenn eine Kultur meint, sie sei die eine wesentliche Kultur, der sich alle anderen unterordnen müssten, dann würde dies bedeuten, dass wir alle diese Beine gewaltsam zusammenbinden und dann, infolge einer Verletzung des Paradigmas des Lebendigen, alle gemeinsam stolpern und fallen werden.

\section{Die Zukunft hängt von uns ab}

Die Wirklichkeit ist ein nicht-auftrennbares, immaterielles Beziehungsgefüge, eine Art „Erwartungsfeld“ für zukünftig mögliche energetisch-materielle Manifestationen. Die Zukunft ist dabei wesentlich offen, was heißen soll, dass sie nicht ganz beliebig offen, sondern durch gewisse allgemeine Bedingungen eingeengt ist, die mit den sogenannten Erhaltungssätzen zusammenhängen und aus Symmetrieeigenschaften der Dynamik resultieren. Hierzu gehört insbesondere die Erhaltung der Energie, welche die „Masse“, nach Einstein, als eine konzentrierte Form der Energie einschließt, doch auch die Erhaltung der elektrischen Ladung und anderer. Sie sorgen dafür, dass bei der Mittelung im Großen überhaupt Eigenschaften, die Kenngrößen der klassischen Physik, übrig bleiben und greifbar werden. Unbelebtes und Belebtes sind nicht mehr grundsätzlich unterschiedlich, sondern erscheinen als statisch stabile bzw. dynamisch stabilisierte statisch instabile Agglomerate, besser: Artikulationen, geformte Teilhabende des Ganz-Einen. Mit der wichtigen Konsequenz: Mensch und Natur sind, wie alles, bei dieser Sichtweise prinzipiell nicht getrennt. Das bedeutet nicht, dass sich im Großen nicht qualitative Unterschiede herausbilden können, wie insbesondere die Möglichkeit kreativer Gestaltung im Rahmen der bedingt offenen Zukunft. Die prinzipielle Offenheit der Zukunft hat wesentliche Folgen für unser Verständnis der Welt, ihrer Entwicklung und unserer Beziehung zu ihr. Wie stellen sich die Wissenschaftler der klassischen Physik den Anfang der Welt vor? Am Anfang war ein „Big Bang“. In diesem Urknall muss die ganze Wirklichkeit angelegt sein, alles, was die Forscher über ihre auf etwa 15 Milliarden Jahre bezifferte Vergangenheit durch ihre Beobachtungen herausgefunden haben oder wenigstens vermuten, und alles, was je in Zukunft passieren wird. Alles muss am Anfang eingebaut sein, nichts darf vergessen werden. Alles, was je geschieht, ist einfach nur eine Entfaltung dessen, was schon im Grunde am Anfang angelegt ist. Das ist in seiner Starrheit nicht sehr befriedigend. Die neue Auffassung hingegen vertritt die Meinung: Die Schöpfung ist nicht abgeschlossen, sie ereignet sich in jedem Augenblick neu und wir sind alle als Teilhabende eines nicht-auftrennbaren Kosmos am fortlaufenden Schöpfungsprozess beteiligt.

Es ist ganz wichtig, dass wir uns nicht als Teile, sondern als Teilhabende dieses Kosmos erfahren, der sinngemäß die Beziehung und nicht das Dingliche betont, und es deshalb auf uns alle ankommt. Wir sind Mitschöpfer. Die zukünftige Entwicklung hängt von uns ab. Wir können selbstverständlich die Welt nicht beliebig ändern, aber wir sollten wissen, dass wir mit unseren Entscheidungen auch immer zum Gesamten beitragen. 


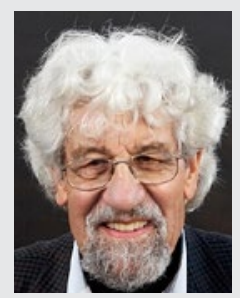

Prof. Dr. Hans-Peter Emil Dürr

ist deutscher Physiker. Bis Herbst 1997 war Dürr Direktor am Max-PlanckInstitut für Physik (Werner-Heisenberg-Institut) in München. Seit 2004 ist er Träger des Großen Bundesverdienstkreuzes. 